\title{
Advances in Miniaturized Instruments for Genomics
}

\author{
Cihun-Siyong Alex Gong ${ }^{1,2}$ and Kin Fong $\mathrm{Lei}^{\mathbf{3 , 4}}$ \\ ${ }^{1}$ Department of Electrical Engineering, School of Electrical and Computer Engineering, College of Engineering, \\ Chang Gung University, Taoyuan 333, Taiwan \\ ${ }^{2}$ Portable Energy System Group, Green Technology Research Center, College of Engineering, \\ Chang Gung University, Taoyuan 333, Taiwan \\ ${ }^{3}$ Graduate Institute of Medical Mechatronics, Chang Gung University, Taoyuan 333, Taiwan \\ ${ }^{4}$ Department of Mechanical Engineering, Chang Gung University, Taoyuan 333, Taiwan \\ Correspondence should be addressed to Kin Fong Lei; kflei@mail.cgu.edu.tw
}

Received 18 December 2013; Revised 21 January 2014; Accepted 30 January 2014; Published 29 May 2014

Academic Editor: Tzu-Chen Yen

Copyright (c) 2014 C.-S. A. Gong and K. F. Lei. This is an open access article distributed under the Creative Commons Attribution License, which permits unrestricted use, distribution, and reproduction in any medium, provided the original work is properly cited.

In recent years, a lot of demonstrations of the miniaturized instruments were reported for genomic applications. They provided the advantages of miniaturization, automation, sensitivity, and specificity for the development of point-of-care diagnostics. The aim of this paper is to report on recent developments on miniaturized instruments for genomic applications. Based on the mature development of microfabrication, microfluidic systems have been demonstrated for various genomic detections. Since one of the objectives of miniaturized instruments is for the development of point-of-care device, impedimetric detection is found to be a promising technique for this purpose. An in-depth discussion of the impedimetric circuits and systems will be included to provide total consideration of the miniaturized instruments and their potential application towards real-time portable imaging in the "omics" era. The current excellent demonstrations suggest a solid foundation for the development of practical and widespread pointof-care genomic diagnostic devices.

\section{Introduction}

Genomics has become an important part of our life since its name was established in the latter half of the twentieth century. It was derived from genetics which includes "classic" and "molecular" as a whole. Polymer chain reaction (PCR) technique is a gold standard for clinical genomic diagnosis. Normally, the concentration of genomic sample is too low for generating detectable signal. PCR can amplify a few copies of DNA to millions of copies of a particular DNA sequence. The technique relies on thermal cycling, that is, repeated heating and cooling of the reaction, for DNA melting and enzymatic replication of the DNA. Generally, twenty to forty thermal cycle times are involved and they take several hours to complete. Although this technique is sensitive for genomic detection, it is time consuming and labor intensive, limiting the throughput of the diagnosis.
In order to enhance the efficiency of the biological reaction, reduce the usage of reagent and sample, and eliminate the fault by human handling, miniaturized instruments that handle small quantity of fluid, for example, microliter or nanoliter, were proposed for the next generation of the diagnostic equipment. Such instruments are also named as microfluidic systems, lab-on-chip (LOC) devices, biochips, or micrototal-analysis systems ( $\mu$ TAS). Because fluid in small amount is manipulated in microscale environment, one of the important properties is to highly enhance the surface-tovolume ratio of the fluid. For some specific applications, high surface-to-volume ratio can benefit the process efficiency. For example, DNA hybridization in rapid diagnostic device normally involves a solid support for the immobilization of the reactants, that is, probe DNA strands. The counterpart of the reactant, that is, target DNA strands, is introduced to the site for binding reaction. The binding efficiency is 
based on the collision possibility. Because of the reduction in diffusion distance and increase in surface-to-volume ratio in microfluidic environment, the reaction kinetics of DNA strands binding reaction was shown significantly accelerated compared with the conventional microplate technique [15]. That results in greatly improving the response time of the biological reaction and the sensitivity of the biological detection. Microfluidic system is often interpreted to a miniaturized version of bioanalytical laboratory. It can perform the entire analytical protocol, such as sample preparation, reagent application, biological reaction, and detection automatically to eliminate the handling fault. Since microfluidic system is a miniaturized instrument, portability is realizable for the point-of-care diagnostic applications.

The aim of this paper is to report on recent developments on miniaturized instruments for genomic applications. An overview of microfluidic systems and their demonstrations for genomic diagnosis will be discussed. Moreover, impedimetric detection is found to be a promising technique for point-of-care genomic detection because the impedimetric signal can easily be analyzed by miniaturized electrical circuits. In-depth discussion of the consideration and review of impedimetric circuits and systems will also be included in this article.

\section{Miniaturized Instruments: Microfluidic Systems}

In the past decade, development of the microfluidic technology becomes intensive and many research articles are available [6-11]. The fabrication of microfluidic systems was originally based on the silicon fabrication technology from semiconductor and microelectromechanical systems (MEMS). Silicon microfabrication is well established but silicon material is not optically transparent and is electrically conductive. Hence, it is not appropriate for the biomedical applications. For example, the microfluidic system for cell culture is required to be transparent for continuous optical monitoring of cell morphology. Moreover, microfluidic system for glucose detection is based on electrochemical reaction which needs insulated substrate for measurement. Therefore, silicon may not be an appropriate material when optical and electrochemical detections are adopted in the microfluidic systems. Therefore, glass and polymeric materials were used because they are less expensive, optically transparent, and not electrically conductive. Specific fabrication technologies for microfluidic systems were introduced, such as soft lithography, hot embossing, and substrate bonding techniques. Soft lithography represents a nonphotolithographic strategy based on self-assembly and replica molding for carrying out micro- and nanofabrication [12]. An elastomeric stamp with patterned relief structures on its surface is used to generate patterns and structures with feature sizes ranging from $30 \mathrm{~nm}$ to $100 \mu \mathrm{m}$. It provides a convenient, effective, and low-cost method for the formation and manufacturing of micro- and nanostructures. Hot embossing technique is for mass production of plastic microcomponents [13]. A mold with microstructures is pressed into a thermoplastic polymer film heated beyond its glass transition temperature under vacuum. After cooling down, the microstructures can be transferred from the mold to the polymer film. To fabricate a functional microfluidic system, substrate bonding is an important process and adhesion between substrates is a problem of great practical concern. Thermal compression, ultrasonic, or gluing by application of either epoxy or methanol may induce global and localized geometric deformation of the substrates or leave an interfacial layer with significant thickness variation. Therefore, special bonding processes for glass and polymeric materials have been developed for fabricating microfluidic systems [1417]. Localized welding of polymeric materials embedded metal films located between the desired bond surfaces by microwave energy has been developed [15]. The bonding can be achieved with $10 \mathrm{~W}$ microwave power in $120 \mathrm{~s}$.

Based on the mature development of the fabrication technology, a board spectrum of biological analytical applications has been demonstrated using microfluidic systems, such as DNA analysis [1, 18-24], immunoassay [25-31], and cell analysis [32-38]. For example, immunoassay on compact disc (CD) has been demonstrated and fluids in $\mathrm{CD}$ were manipulated by the centrifugal forces controlled by the rotational speed of the CD [30]. Illustration and photograph of the CD-based microfluidic system are shown in Figure 1. High throughput screening of analytes could be realized by simultaneous functions in parallel layouts on the CD. Enzyme-linked immunosorbent assay (ELISA) was demonstrated on this CD-based platform. Another example is to construct a microfluidic chip for real-time and noninvasive impedimetric monitoring of cell proliferation and chemosensitivity in three-dimensional (3D) cell culture construct, as shown in Figure 2 [37]. Human oral cancer cells (OEC-M1) were encapsulated in 3D agarose scaffold and cultured in a miniaturized chamber under perfusion of tested substance. This setting provides a more in vitro physiologically relevant microenvironment to better mimic the complex in vivo microenvironment. These excellent developments showed the capability of microfluidic system for performing complex analytical applications. Commercial possibility is obvious because the microfluidic system can provide a total solution of biological analysis from the sample application to the display of the analysis results. Point-ofcare diagnostic applications can be realized based on the advantages of miniaturization, integration, and automation of the microfluidic system.

\section{Integrated Microfluidic Genomic Systems}

Microfluidic systems have been also applied to the genomic applications. System integrated with microchannels, heaters, temperature sensors, and fluorescence detectors was fabricated for the functions of capturing DNA, mixing solutions, amplifying DNA, and separating and detecting of those products [20]. These complicated operations could be performed on a single glass and silicon substrate. Strand displacement amplification experiment was conducted and showed that the specific target DNA was successfully amplified and detected. 


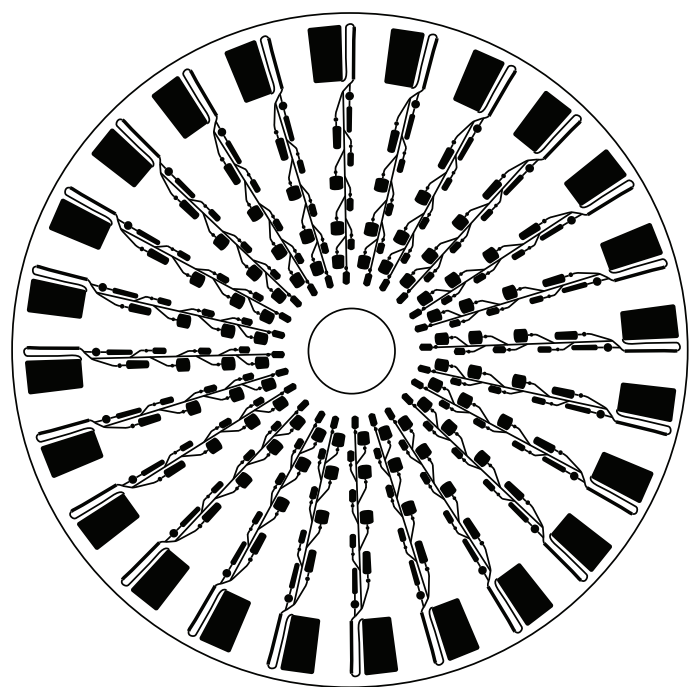

(a)

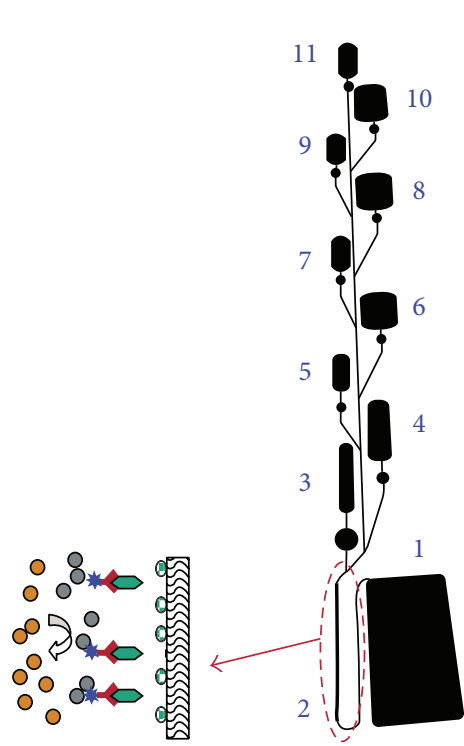

(b)

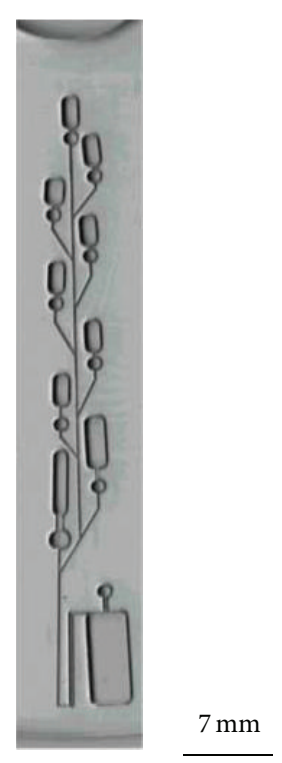

(c)

FIGURE 1: Schematics of (a) a CD-ELISA design with 24 sets of assays, (b) a single assay, and (c) photo of a single assay. Copyright 2004. Reprinted from [30] with permission from the American Chemistry Society.

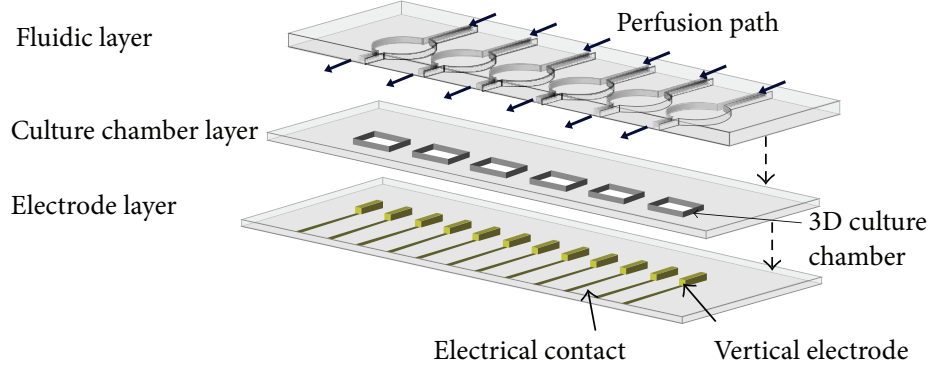

(a)

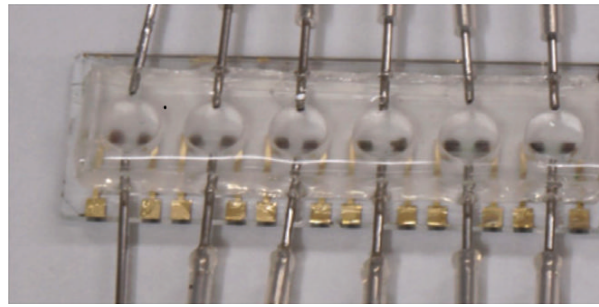

(b)

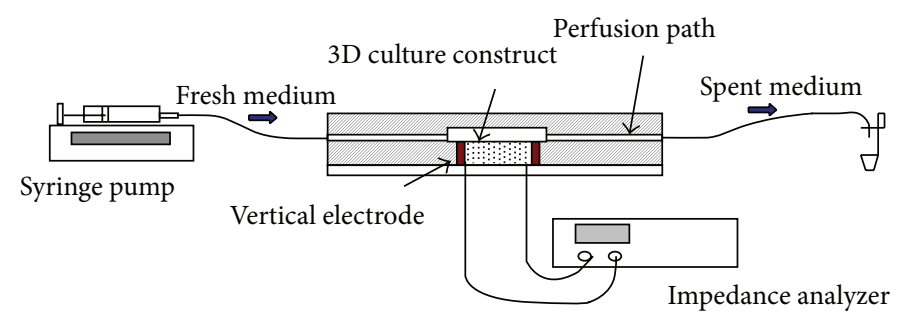

(c)

Figure 2: (a) Design of the microfluidic chip. (b) Photograph of the microfluidic chip. (c) Illustration of the experimental setup of the perfusion 3D cell culture incorporated with on-site impedance measurement. Copyright 2014. Reprinted from [37] with permission from the Elsevier.

Moreover, PCR is a widely used technique in biological applications and was implemented on a microfluidic system, as illustrated in Figure 3 [39]. The PCR was achieved by introducing the reactant droplet into the inlet. Three reaction chambers, respectively, stabled at $90^{\circ} \mathrm{C}, 72^{\circ} \mathrm{C}$, and $55^{\circ} \mathrm{C}$ were integrated in a chip and droplet was driven back and forth by three piezoelectric micropumps between these three reaction chambers. After 20-30 thermal cycles, the PCR products were pumped into the reservoir to be collected and analyzed by gel electrophoresis. Also, an electrokinetically controlled DNA hybridization microfluidic chip has been demonstrated and can perform all processes from sample dispensing to hybridization detection within 5 minutes [1]. The chip consisted of a PDMS upper substrate and a lower glass substrate that served as a substrate for the hybridization array, as shown in Figure 4. The design of the chip was an H-type channel structure containing immobilized single-stranded oligonucleotide probes. The electroosmotic pumping could 


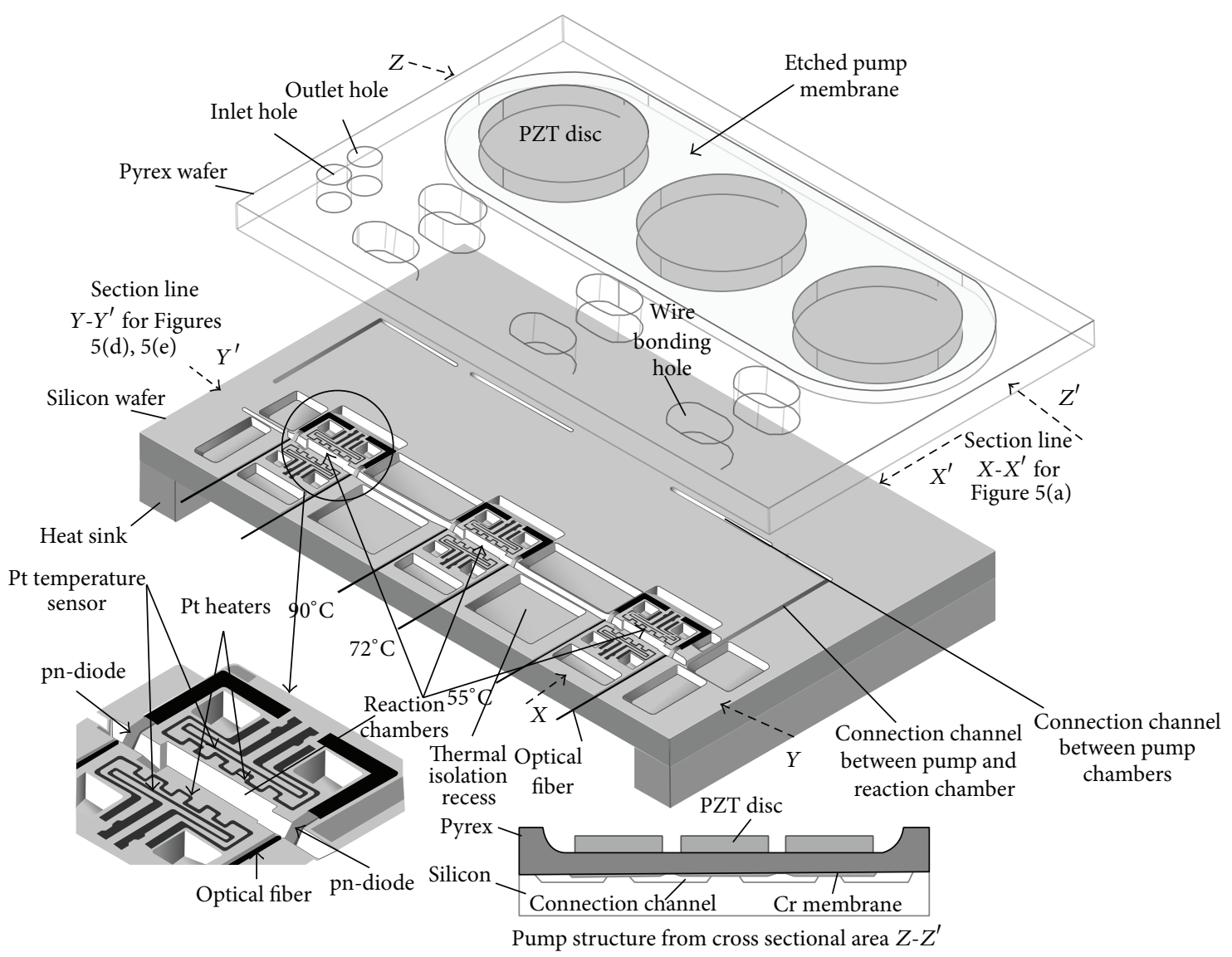

FIGURE 3: Schematic of the pump PCR chip. For simplification, the upper glass wafer and the lower silicon wafer are illustrated apart, although in the actual device both wafers are connected by anodic bonding. The lower left insert figure shows an expanded view of the reaction chamber and the lower right insert shows the cross-section of the micropump. Copyright 2003. Reprinted from [39] with permission from IOP Publishing Ltd.

dispense the controlled samples of nanoliter volume directly to the hybridization array and remove nonspecific adsorption. Hybridization, washing, and scanning procedures can be conducted simultaneously. Detection levels as low as $50 \mathrm{pM}$ were recorded using an epifluorescence microscope.

\section{Impedimetric Detection of Genomic Signal}

In conventional genomic detection, optical measurement, for example, fluorescent labeling technique, was utilized to quantify the genomic activity, for example, DNA hybridization and PCR product. But this measurement technique is time consuming and labor intensive. Alternatively, impedimetric detection was proposed to be one of the promising techniques to quantify biological activity in the microfluidic systems. The detection results are represented by electrical signals which can easily interface with miniaturized instruments. For example, electrical detection of DNA hybridization using electrochemical impedance spectroscopy (EIS) was demonstrated [40]. Results showed a $25 \%$ increase of impedance for double-stranded DNA on gold electrode compared with the same electrode with immobilized single-stranded DNA. Another example showed that the DNA hybridization could be detected by the resistance change across the electrode $[41,42]$. DNA hybridization on a pair of electrodes was indicated by gold nanoparticles and the gold nanoparticles were physically amplified to a silver conductive layer on the electrode. The hybridization result could be measured by the conductivity changes across the electrode. These demonstrated showed an alternative method for detecting the genomic signal. For the application of cell proliferation study, the entire process requires a long period of time and in a controlled environment. It is more preferable to perform in a bench-top system. However, a miniaturized and portable device is more preferable for the on-site rapid diagnostic application. The combination of microfluidic and impedimetric technologies would be suitable for such a specific application.

\section{Impedimetric Foundation}

As mentioned, the impedimetric method provides a versatile way that can be used for many biological applications including the quantification of genomic activity and the noninvasive monitoring of cell proliferation and chemosensitivity with a microfluidic chip. The underlying principle of the technique 


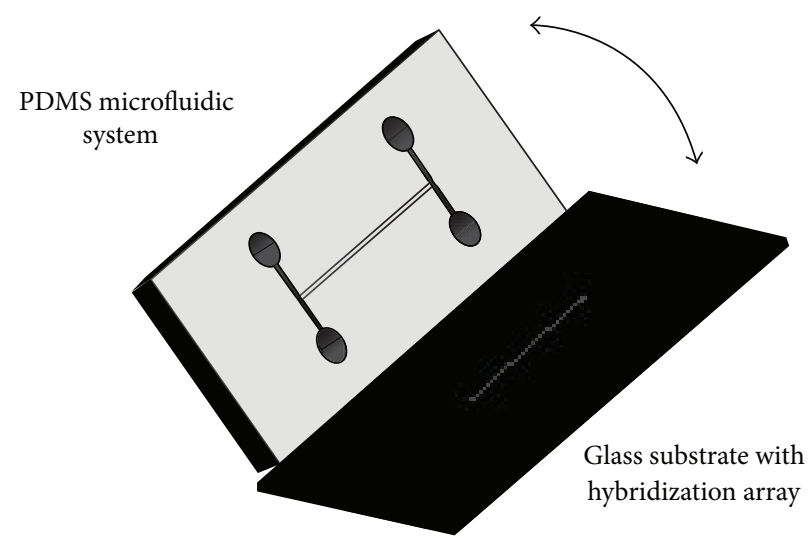

(a)

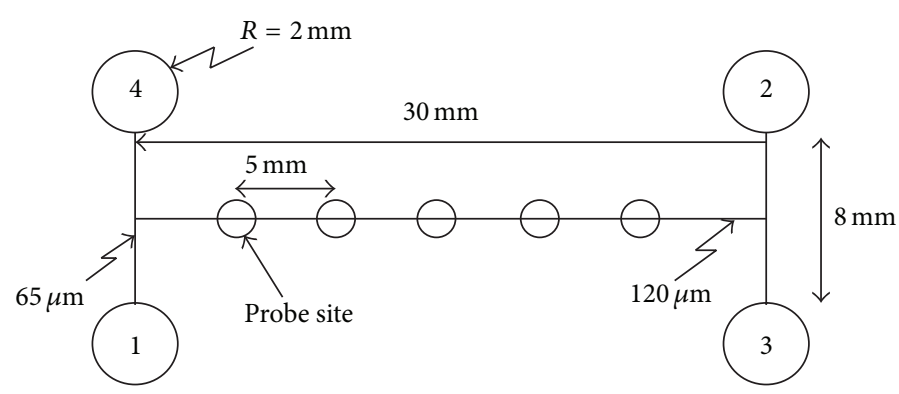

(b)

FIGURE 4: (a) Assembly procedure for PDMS fluidics and immobilized hybridization array. (b) H-type channel structure for DNA hybridization chip: (1) sample port, (2) auxiliary port, (3) buffer port, and (4) wash port. Copyright 2004. Reprinted from [1] with permission from the American Chemical Society.

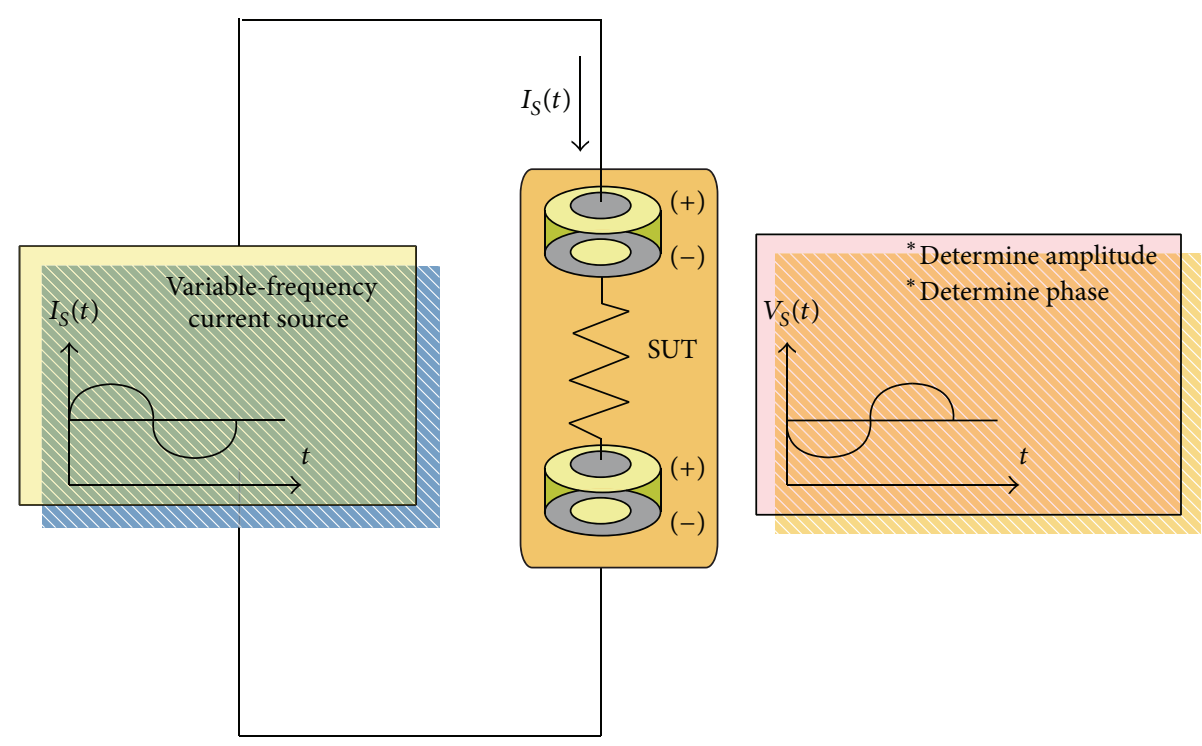

FIGURE 5: The basic model of equivalent circuit used to elaborate on the relation of sample under test (SUT) to the generated current source and resulting voltage response.

can be explained from Figure 5. Let us assume, for the sake of simplicity, that the sample under test (SUT) consists only of a resistor that is connected with two electrodes. The upper electrode is commonly called "anode" or "working electrode." The lower electrode is called "auxiliary electrode" functioning as "cathode." To understand SUT, an active alternating current $(\mathrm{AC}), I_{S}(t)$, is generated and injected into the close loop. The resulting voltage drop across the two electrodes can be measured to derive the resistance of SUT by means of Ohm's law, provided that both the two electrodes have zero voltage drop. When the equivalent circuit of electrode becomes a complex number, as the combination shown in Figure 6(a), variable-frequency current source is required to draw socalled "Nyquist plot" (Figure 6(b)) [43]. The model shown in Figure 6(a) is based on electrochemical point of view. It is consisted of an ohmic resistance $R_{o}$ stemming from the solution resistance and electrode geometry, a charge transfer resistance $R_{\mathrm{ct}}$ stemming from the charge transfer between the interface of electrode and electrolyte, an electric double-layer capacitance $C_{d}$ stemming from placing a large-area charge in the electrolyte in proximity to that on the porous electrodes at the medium-frequency region, a Warburg impedance $Z_{w}$ representing the mobility of the internal ions resulting from the diffusion and migration at the low-frequency region, and an electrode inductance $L_{d}$ stemming from reduction in the penetration depth of the ions at the high-frequency region, respectively $[43,44]$. As a matter of fact, with regard to the ohmic resistance of SUT, an electrode immersed into an electrolyte creates a potential that is related to the oxidationreduction concentration, according to the Nernst law [44]. The corresponding potentials cancel out as long as the two electrodes are the same. Unfortunately, this would never 


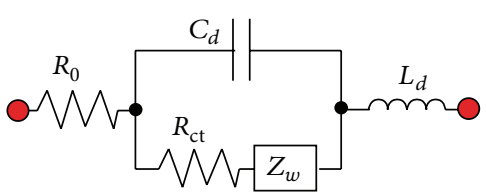

(a)

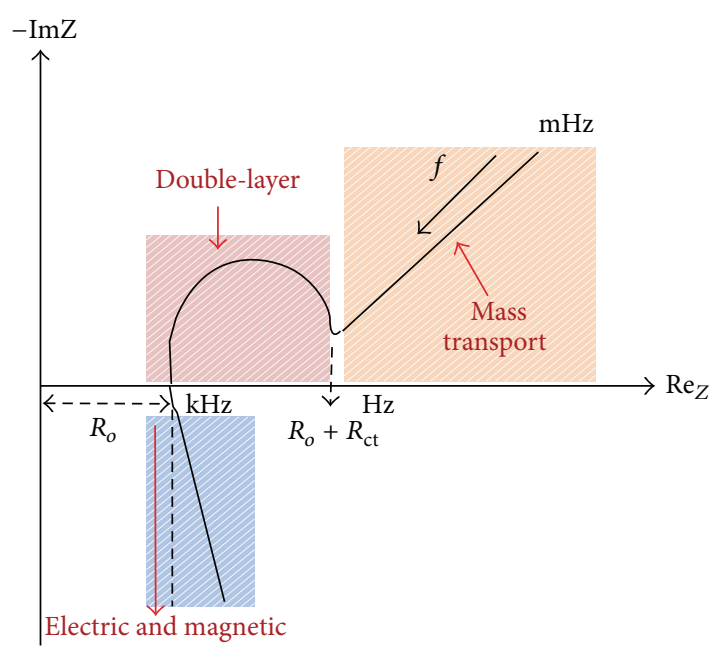

(b)

Figure 6: (a) Schematic of the equivalent circuit of electrode in an electrochemical point of view. (b) The so-called Nyquist plot showing the characteristic of frequency response versus the decomposition of impedance.

happen and a potential difference of a few millivolts would always exist between SUT and either of the electrodes [44].

\section{Impedimetric Consideration}

There have been several technically sound circuits and systems demonstrated in the literature to implement the impedimetric method so far [44-49]. They are similar to a coherent demodulation technique demonstrated in Figure 7, where a four-electrode method was adopted [45]. The impedance sensing method shown in Figure 5 is premised on the assumption that both working and auxiliary electrodes have resistance value of "zero." However, as mentioned, this would never be the case and there exist voltage drops of them in the close loop as soon as an electrical current flows through, turning out that certain inversion formula is unavoidable for derivation of the ohmic resistance of SUT. This may be taxing on postprocessing and result in incapability of real-time impedance monitoring. By taking the advantages of the advances in modern semiconductor technologies, an amplifier with ultrahigh input impedance (almost open circuit) can be readily available. In addition, differential sensing is always a better choice than the singleended counterpart as a result of better noise immunity $[46,50]$. These form the foundation of the architecture shown in Figure 7.

\section{Circuits and Systems for Bioimpedance Measurement}

Referring to Figure 7, in addition to the necessary electrodes Ze1 and Ze4 to form a loop, two additional electrodes Ze2 and Ze 3 were added and combined with the instrumentation amplifier (IA) whose common-mode rejection ratio (CMRR)

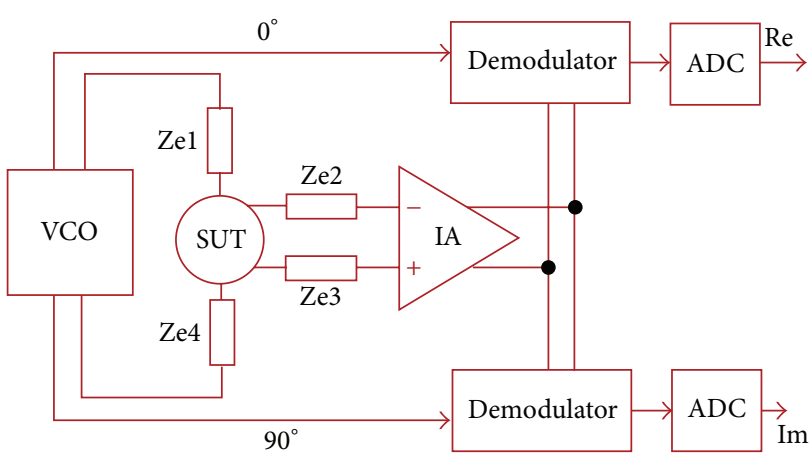

FIGURE 7: Impedance sensing architecture presented in [45], with which the four-electrode method accompanies, demonstrating the coherent demodulation technique.

is significantly improved as compared with the ordinary counterpart $[45,46]$. The variable-frequency sinusoidal current used for sensing was generated by a dedicated voltagecontrolled oscillator (VCO). Thanks to the high-impedance feature of the amplifier, there was no current flowing through $\mathrm{Ze} 2$ and Ze3. As a result, the sensed voltage drop across SUT has predetermined current and therefore can be used to represent the impedance of SUT. A system with this kind of 4-electrode configuration is also known as a system using "tetrapolar method" [48].

To decompose the complex number of impedance, two orthogonal AC signals are required in the coherent demodulation, based on the Euler's formula to represent a periodic signal using a combination of sine and cosine. The AC signals were generated in the same VCO to reduce the system complexity and save the implementation cost. The Demodulator circuits functioned as "mixer" and their outputs were quantized by the dedicated analog-to-digital 


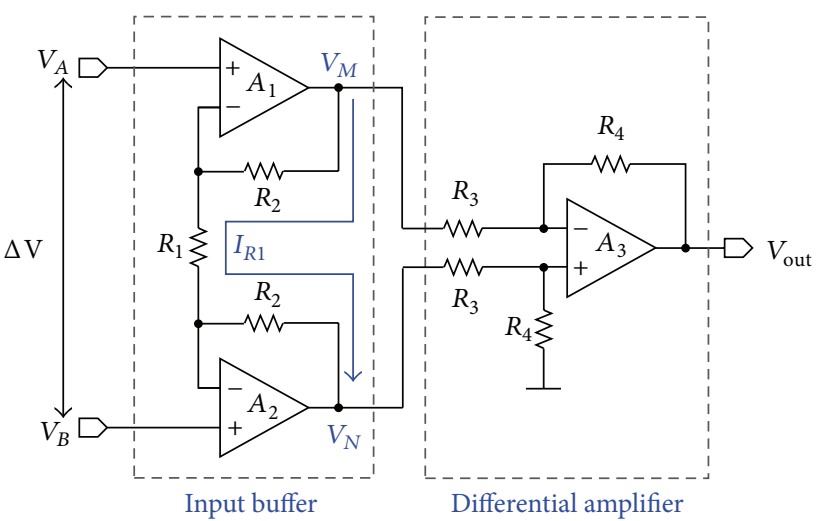

(a)

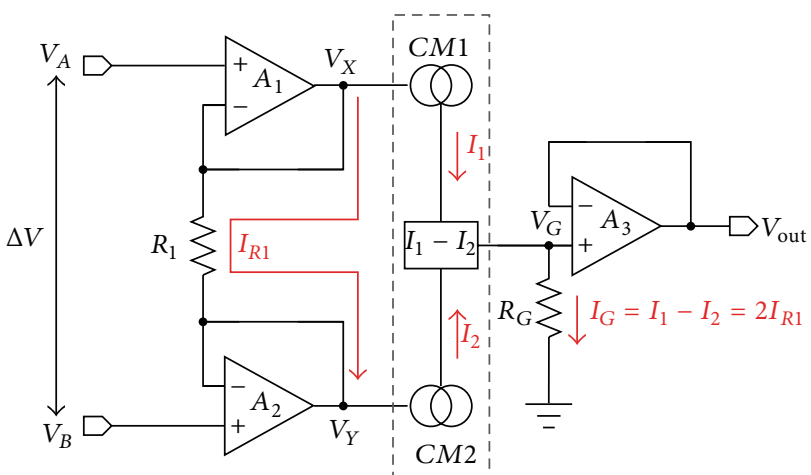

(b)

FIGURE 8: (a) The circuit schematic of conventional instrumentation amplifier (IA) in [50]. (b) The circuit schematic of improved counterpart in [51].

converters (ADCs). The real part $(\mathrm{Re})$ and imaginary part (Im) can be used to draw Nyquist plot for impedance analysis. It should be noticed that, practically, there still exists certain electric potential difference between $\mathrm{Ze} 2$ and $\mathrm{Ze} 3$ in spite of the zero current at the inputs of IA, and due to that Ze2 and Ze3 could not be identical. As a result, high CMRR is necessary to reject the potential difference of IA, resulting in design challenge. The major bottleneck in implementation is the matching of resistors involved in the commonly adopted IA structure shown in Figure 8(a) [50] where the output of IA can be expressed as

$$
V_{\text {out }}=-\frac{R_{4}}{R_{3}}\left(1+\frac{2 R_{2}}{R_{1}}\right)\left(V_{A}-V_{B}\right)=K_{1}\left(V_{A}-V_{B}\right) .
$$

Achieving sufficient resistive matching between $R_{4}$ and $R_{3}$ (or $R_{2}$ and $R_{1}$ ) to obtain high CMRR relies on post-ICfabrication trimming, which is cost ineffective and difficult to fulfill miniaturization in practice. As a result, the design shown in Figure 8(b) was proposed [51]. The most significant feature of the design is that it requires only two resistors. $A_{1}$ and $A_{2}$ form source followers as conventional, thereby forcing $V_{A}=V_{X}$ and $V_{B}=V_{Y}$. The current flowing out of $A_{1}$ and that of $A_{2}$ are equal, but they have opposite polarities. By using a current subtractor, marked in the dotted line, one can obtain $I_{G}=2 I_{R 1}$; hence the output of IA becomes

$$
V_{\text {out }}=\frac{2 R_{G}}{R_{1}}\left(V_{A}-V_{B}\right)=K_{2}\left(V_{A}-V_{B}\right) .
$$

This circuit structure successfully alleviates the impact of mismatched resistance, achieving both high CMRR and miniaturization at the cost of increased power consumption as compared with that shown in Figure 8(a). High CMRR can also be attained by means of considerably increased differential gain. Unfortunately, the energy efficiency of system is further compromised.

An often overlooked factor in correct impedance monitoring is that the electrode-referred DC offset (ERDO) limits the available CMRR, affecting the operation of IA and degrading overall performance no matter how good the following circuits and systems can be. Two renowned techniques have been proposed so far to cancel ERDO. A technique called "autozeroing" is shown in Figure 9(a) [52]. It uses three switches to cancel ERDO, $V_{O S}$. When $\phi$ is at a logic-"high" level, the amplifier involved samples $V_{O S}$ and store them on $C_{S}$. Assuming that the open-loop gain of amplifier is $A$, the voltage on $C_{S}$ will be $V_{O S} \cdot(A /(1+A))$ after settling. When $\phi$ becomes a logic- "low" level, $V_{O S}$ of previous state will be subtracted from $V_{i}$ which is superimposedwith current $V_{O S}$ which will be with the same value as that of previous state, resulting in a considerably decreased ERDO of $V_{O S} \cdot(1 /(1+A))$ present at the positive terminal of amplifier. The autozeroing technique can also effectively reduce the "Flicker" noise of modern semiconductor process but comes with a penalty of high-frequency interference stemming from the sampling clocks of the switches. Its major drawback is the wide bandwidth of amplifier as a result of the voltage settling on $C_{S}$.

Another efficient candidate is the work shown in Figure $9(\mathrm{~b})$ where $V_{\text {ip }}$ and $V_{\text {in }}$ can be connected with Ze2 and $\mathrm{Ze} 3$, respectively. The circuit serves as a preamplifier located between the electrodes and IA to "continuously" remove ERDO [53]. Here we use single differential circuit configuration to detail its advantage but then it can turn into its fully differential counterpart to provide two output terminals to IA. The design embodies the AC coupling to reject ERDO in order to make itself free from malfunction as a result of the saturation. The low-frequency cutoff of the high-pass filter formed by the $R_{2}-C_{2}$ network can be adjusted through their time constant. The low-pass corner frequency can be adjusted by the time constant of the lumped impedance at the output of the preamplifier and $C_{L}$. Owing to the low frequencies required by the impedance measurement, an extremely large $R_{2}-C_{2}$ time constant is unavoidable. As a result, a pseudoresistor configuration shown in Figure 9(c) can be adopted [53]. The pseudoresistor operates the transistors involved at "subthreshold" operation to achieve a large equivalent resistance value that is almost impossible to be 


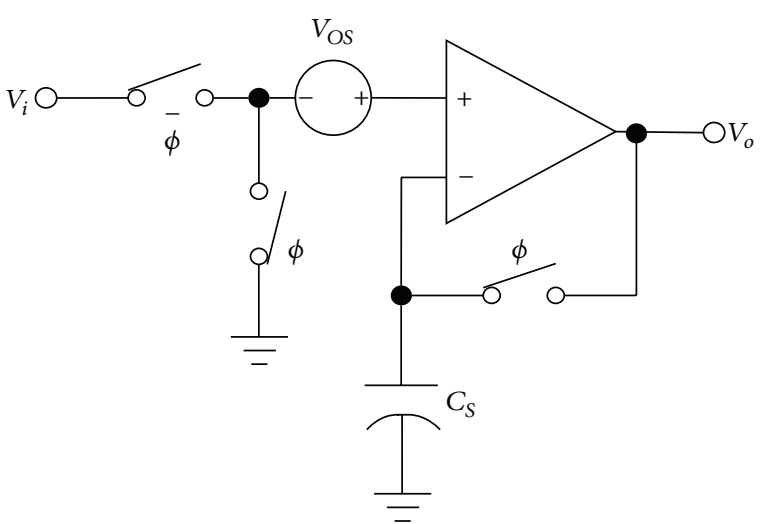

(a)

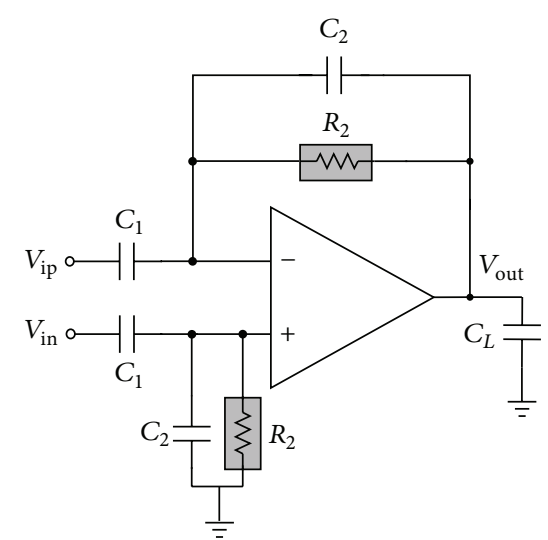

(b)

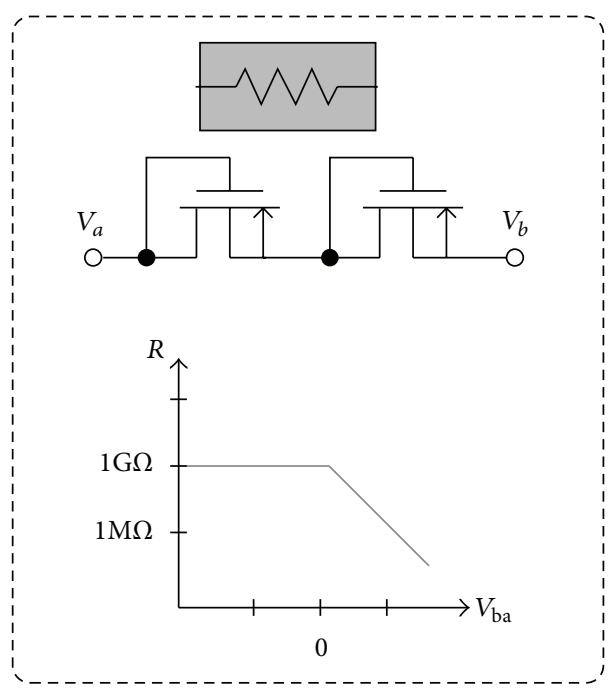

(c)

Figure 9: (a) The autozeroing technique [52]. (b) The AC-coupled technique [53]. (c) The pseudoresistor technique [53].

realized on the basis of "on-chip" miniaturization. The closedloop midband gain of the preamplifier can be determined by $C_{1} / C_{2}$.

It should also be noticed that the accuracy of current of measurement, signals generated from VCO for the demodulation and how accurate their frequencies and phases can be achieved by the system shown in Figure 7, will also affect the final outcome dramatically. The frequencies and phases can be adjusted and finely tuned by means of a phaselocked loops with a precise reference frequency [54]. Such a frequency can be generated from an electronic circuit containing a mechanically resonant vibrating crystal (so-called crystal oscillator) [54]. Precise current of measuring SUT can be obtained through the use of a "current mirror" with sufficiently high output impedance. Modern semiconductor technologies offer many well developed and miniaturized circuit topologies to achieve such a goal $[55,56]$.

To advance miniaturization, the architecture shown in Figure 7 could be further improved as the complexityreduced alternative shown in Figure 10 [48]. The architecture, which is called synchronous sampling, has mainly two most significant features: (a) removal of IA and (b) representing final results in pulse-width modulation (PWM) (using a one-bit $\mathrm{ADC}$ ). The elements $Z_{\mathrm{EA}}$ to $Z_{\mathrm{ED}}$ correspond to the impedance of the four electrodes and the media were modeled by the elements $Z_{\mathrm{MA}}$ to $Z_{\mathrm{MD}}$. Each of these impedances has real and imaginary components associated with the conductivity and dielectric properties of the media, respectively [48]. The voltage on the negative terminal of OTA BIAS will be forced to become the reference voltage $V_{\text {ref }}$ which was set to halve the supply voltage and was used as the "ground" in the analog circuits involved, thanks to the high open-loop gains of the amplifiers achieving the "virtual short." It turns out to be reducing the loss in the parasitic elements and avoiding the need for IA and the differential AC-coupled inputs. The demodulated results, followed by the low-pass filter (LPF), were compared with $V_{\text {ref }}$ to obtain a PWM waveform that is easy to be transmitted wirelessly without parallel-serial converter commonly seen at the output of ADC for serial link. This architecture avoids two demodulation channels by incorporating a sampling mechanism using the proper sampling times. 


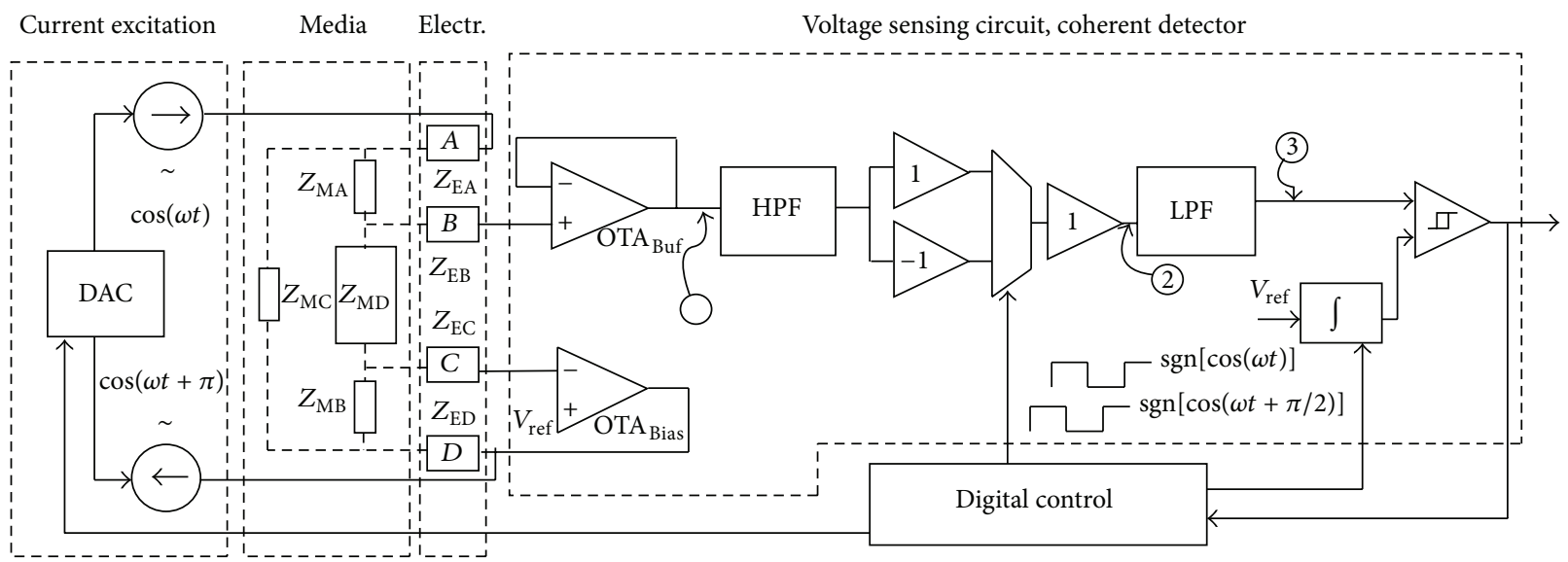

FIGURE 10: Synchronous sampling impedance sensing architecture. Copyright 2009. Reprinted from [48] with permission from Elsevier.

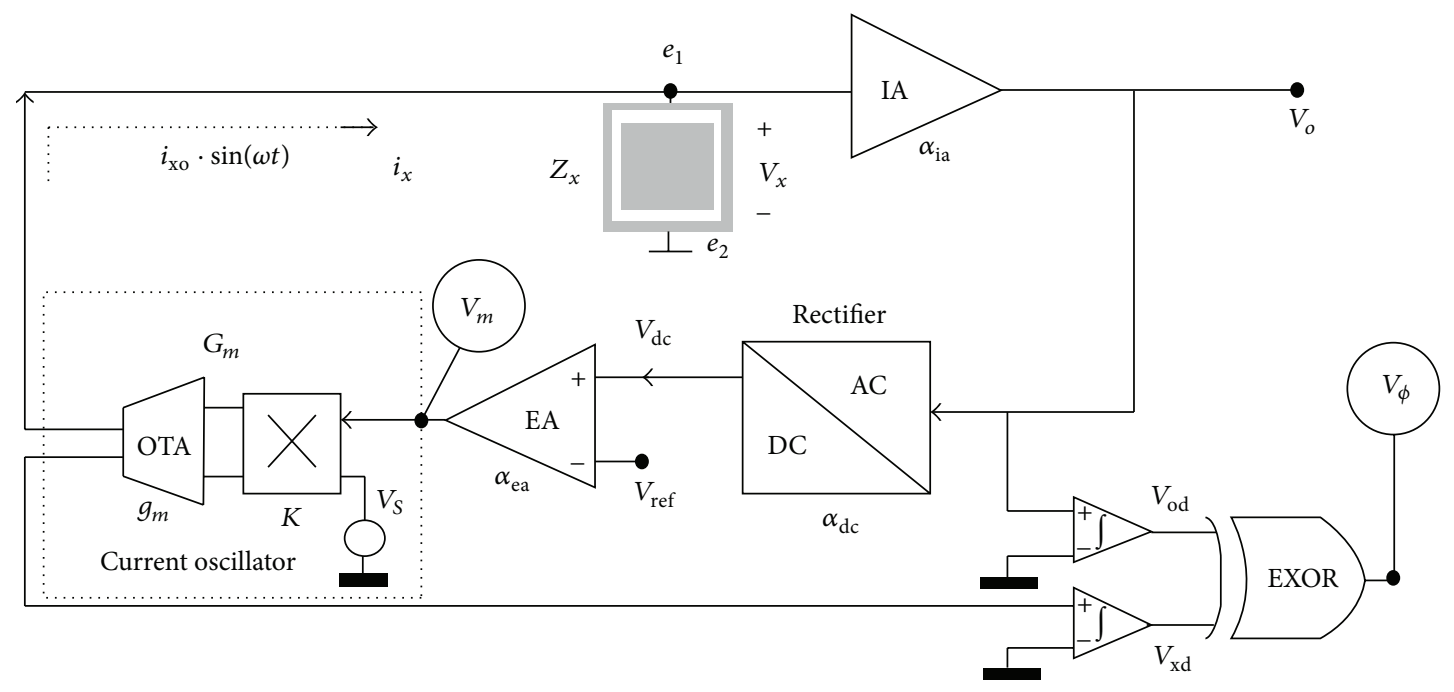

FIGURE 11: The closed-loop impedance sensing architecture. Copyright 2010. Reprinted from [49] with permission from Elsevier.

Recently, a closed-loop architecture shown in Figure 11 was proposed [49]. Despite the same theory principle for impedance measurement, its target used to determine the final outcome is unlike the two representative architectures shown in Figures 7 and 10. In this design, the resulting voltage across the impedance under test (ZUT) including SUT will be confined to a predetermined amount using the error amplifier EA with the given reference voltage $V_{\text {ref }}$ at its input. This will help to operate the electrodes involved in a linear and predictable region. The generated AC current $i_{X}$ flowing through ZUT can be controlled timely as a result of the feedback loop at $V_{\mathrm{O}}$. Because the transconductance of OTA $\left(g_{m}\right)$ can be deduced during design and measurement phases and both the multiplication factor $K$ and signal source $V_{S}$ can be given, $i_{X}$ can be obtained, provided that $V_{m}$ is available after being monitored. With $V_{X}$ and $i_{X}$, the impedance "magnitude" of ZUT can be measured. The impedance "phase" can be measured by comparing the digitized results $V_{\text {od }}$ and $V_{\text {xd }}$ of $V_{O}$ and the output of OTA to each other.

Although the architecture shown in Figure 11 provides a good candidate to achieve not only an operation taking the contribution of electrodes into account but also a safe measurement with a decent accuracy as compared with others demonstrated in the literature, its overall performance is governed by the bandwidth, open-loop gain, input offset, and CMRR of amplifier, similar to its counterparts. However, high-gain, wide-bandwidth, low-offset, and high-CMRR amplifier consumes considerable power consumption, which goes against portability requiring miniaturization. In a nutshell, it has been believed that the performance of analog front end is of primary importance for the precise measurement of impedimetric system. The miniaturization effort involves making trade-offs among different aspects of mixed-signal (analog and digital) circuit design. The technical strategies 


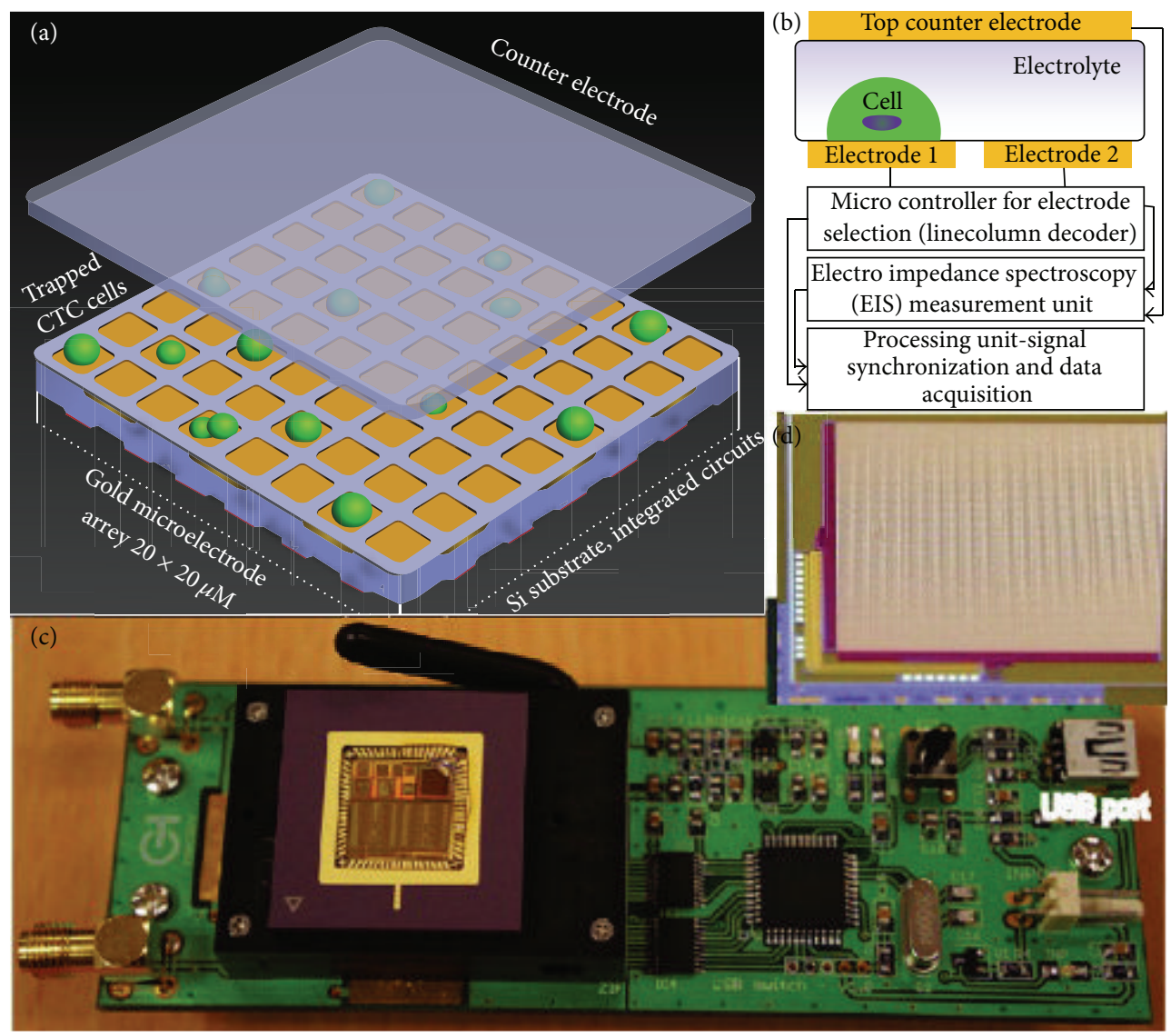

FIGURE 12: CMOS based sensor array for cell counting. (a) Schematic of the microelectrode arrays for the cell detection. (b) Illustration of the sensor layout and the addressing scheme employed in the CMOS sensor chip. (c) CMOS chip packaged with a switching PCB. (d) Microphotograph of more than nine thousand electrodes in a single chip. Copyright 2012. Reprinted from [57] with permission from Elsevier.

illustrated with Figures 8 and 9 are by no means the total solutions but have demonstrated that they can be used to effectively deal with the mentioned problems in terms of miniaturization point of view. Last but not least, with regard to some implantable applications where an extremely miniaturized design of real-time impedance monitoring must be fulfilled in limited space to allowing integration to the most degree, the test current of SUT and ZUT could be generated by an electrical stimulator without the dedicated circuit such as VCO, DAC, or current oscillator shown in Figures 7, 10, and 11 , respectively $[56,58,59]$. This turns out to be good for the system on a chip (SoC) in modern semiconductor technologies.

\section{Impedimetric Imaging Instrumentation in Omics}

We have reviewed in detail the technologies regarding miniaturization. One might want to know the relevance between them and "imaging." For the delivery of next-generation therapies, functional characterization of genes using a systematic way is imperative. One of the manners doing this kind of characterization requires downregulation of the expression of specific genes in order to comprehensively study the functions of genes [60]. To this end, the cell-based functional assay has been emerged as one of the powerful tools for acute observation [61]. The cell-based functional assay can be used to acquire the information about the phenotypic effect of targeted "gene knockdown," which is a technique to reduce the expression of one or more of an organism's genes, in a way "incisive" when using RNA interference (RNAi) [62]. However, almost all of the assays are used only for experiencing a rapid onset (i.e., to say "for a given point of time") currently, implying that most of the changes are missed in measurement. In addition, it has been demonstrated that advanced state-of-the-art electronic biosensors with microwell plates should be developed to be able to record impedimetric cell-to-electrode responses in a way "label-free" by means of microelectrodes. The combination of the requirements "continuous monitoring," "impedimetric cell-to-electrode recording," and "bidimensional-space (2D) electrode array manipulation" form the base of newgeneration time-dependent profiling for cell responses. One of the most recent works with respect to the development of impedimetric spectrum platform with different application regarding cell has been demonstrated in Figure 12 where advanced semiconductor process and circuit techniques have 
been employed to advance miniaturized sensing system with light weight and low power in the platform [57]. By displaying the results of $2 \mathrm{D}$ spectrum continuously, real-time impedimetric imaging can be realized to ceaselessly measure the cell status of importance.

\section{Concluding Remarks}

The ever increasing demand in the modern technologies has improved the quality of life. Microfluidic systems have been applied to different genomic applications and showed realizable opportunity for point-of-care diagnostic devices. The advances in circuits and systems have been driving a technical revolution in the microfluidic systems that are essential to the "-omics" era. Impedimetric detection is a promising technique to develop miniaturized measurement equipment. The improved impact on the SoC techniques has enabled sustainable solutions which have been demonstrated so far to be effective to pressing real problems in such a field. Several representative solutions ranging from impedimetric architectures and efficiency-enhanced miniaturized techniques have been discussed in detail in this paper. Many researchers have pursued the ideas of using the techniques they have learned to facilitate interdisciplinary collaborations among SoC design, micromechanical technologies, material science, and biomedical engineering. It is almost impossible to embody miniaturization towards light weight and low power, while at the same time achieving an accurate impedance signal conditioning and the reduced response time without the help of microfabricated and mixed-signal technologies, not to mention the portability. In addition to the applications and advantages mentioned, it can be envisioned that by leveraging the architectures and techniques, low-price and precise early detection of many fatal diseases, such as the cancers, will eventually come true. Despite the strength and importance of impedance measurement system, those prior arts suffer the most from the contamination of electrode. Once the electrodes dip into the sample, nonspecific adsorption of biological components starts to take place. The contamination of electrode is still an open question and is accompanied with distortion of measured impedance spectrum, resulting in observable (inductive) artifact at some frequencies. In order to eliminate the contamination of detection electrodes and reaction chamber, the device is normally designed to be disposable for the rapid diagnostic applications. Moreover, the electrodes are made of noble metals, for example, $\mathrm{Au}$ and $\mathrm{Pt}$, in order to prevent the surface oxidation. The contamination may also be overcome by having a large number of in vitro tests on electrode-sample reactions (redox) as an index of lookup stored in an on-chip memory. This may greatly help differentiate the shifted impedance spectrum from its normal circumstances (through some kinds of algorithms). In conclusion, the microfluidic systems incorporated with impedimetric detection technique provide simple, miniaturized, and sensitive detection of genomic signal. It is believed that these systems can develop practical point-of-care genomic diagnostic devices.

\section{Conflict of Interests}

The authors declare that there is no conflict of interests regarding the publication of this paper.

\section{Acknowledgments}

This study was supported in part by the National Science Council (NSC), Taiwan, under Grant no. NSC 102-2218E-182-003-. The authors would also like to thank funding support from the Chang Gung University, Taiwan, and Chang Gung Memorial Hospital, Taiwan, under Grants nos. UERPD2D0011, CMRPD2C0141, and CMRPD2D0021.

\section{References}

[1] D. Erickson, X. Liu, U. Krull, and D. Li, "Electrokinetically controlled DNA hybridization microfluidic chip enabling rapid target analysis," Analytical Chemistry, vol. 76, no. 24, pp. 7269$7277,2004$.

[2] P. K. Yuen, G. Li, Y. Bao, and U. R. Müller, "Microfluidic devices for fluidic circulation and mixing improve hybridization signal intensity on DNA arrays," Lab on a Chip, vol. 3, no. 1, pp. 46-50, 2003.

[3] R. H. Liu, R. Lenigk, R. L. Druyor-Sanchez, J. Yang, and P. Grodzinski, "Hybridization enhancement using cavitation microstreaming," Analytical Chemistry, vol. 75, no. 8, pp. 19111917, 2003.

[4] M. K. McQuain, K. Seale, J. Peek et al., "Chaotic mixer improves microarray hybridization," Analytical Biochemistry, vol. 325, no. 2, pp. 215-226, 2004.

[5] Y. C. Chung, Y. C. Lin, M. Z. Shiu, and W. N. T. Chang, "Microfluidic chip for fast nucleic acid hybridization," Lab on a Chip, vol. 3, no. 4, pp. 228-233, 2003.

[6] D. R. Reyes, D. Iossifidis, P. A. Auroux, and A. Manz, "Micro total analysis systems. 1. Introduction, theory, and technology," Analytical Chemistry, vol. 74, no. 12, pp. 2623-2636, 2002.

[7] P.-A. Auroux, D. Iossifidis, D. R. Reyes, and A. Manz, "Micro total analysis systems. 2. Analytical standard operations and applications," Analytical Chemistry, vol. 74, no. 12, pp. 2637$2652,2002$.

[8] T. Vilkner, D. Janasek, and A. Manz, "Micro total analysis systems. Recent developments," Analytical Chemistry, vol. 76, no. 12, pp. 3373-3386, 2004.

[9] Y. Zhang and P. Ozdemir, "Microfluidic DNA amplificationa review," Analytica Chimica Acta, vol. 638, no. 2, pp. 115-125, 2009.

[10] A. Bange, H. B. Halsall, and W. R. Heineman, "Microfluidic immunosensor systems," Biosensors and Bioelectronics, vol. 20, no. 12, pp. 2488-2503, 2005.

[11] K. F. Lei, "Microfluidic systems for diagnostic applications: a review," Journal of Laboratory Automation, vol. 17, pp. 330-347, 2012.

[12] Y. Xia and G. M. Whitesides, "Soft lithography," Annual Review of Materials Science, vol. 28, no. 1, pp. 153-184, 1998.

[13] A. Gerlach, G. Knebel, A. E. Guber et al., "Microfabrication of single-use plastic microfluidic devices for high-throughput screening and DNA analysis," Microsystem Technologies, vol. 7, no. 5-6, pp. 265-268, 2002. 
[14] W. W. Y. Chow, K. F. Lei, G. Shi, W. J. Li, and Q. Huang, "Microfluidic channel fabrication by PDMS-interface bonding," Smart Materials and Structures, vol. 15, pp. S112-S116, 2006.

[15] K. F. Lei, S. Ahsan, N. Budraa, W. J. Li, and J. D. Mai, "Microwave bonding of polymer-based substrates for potential encapsulated micro/nanofluidic device fabrication," Sensors and Actuators A: Physical, vol. 114, no. 2-3, pp. 340-346, 2004.

[16] Z. J. Jia, Q. Fang, and Z.-L. Fang, "Bonding of glass microfluidic chips at room temperatures," Analytical Chemistry, vol. 76, no. 18, pp. 5597-5602, 2004.

[17] C. W. Tsao and D. L. DeVoe, "Bonding of thermoplastic polymer microfluidics," Microfluidics and Nanofluidics, vol. 6, no. 1, pp. 1-16, 2009.

[18] K. F. Lei, H. Cheng, K. Y. Choy, and L. M. C. Chow, "Electrokinetic DNA concentration in microsystems," Sensors and Actuators A: Physical, vol. 156, no. 2, pp. 381-387, 2009.

[19] Y. He, M. Tsutsui, C. Fan, M. Taniguchi, and T. Kawai, "Gate manipulation of DNA capture into nanopores," ACS Nano, vol. 5, no. 10, pp. 8391-8397, 2011.

[20] M. A. Burns, B. N. Johnson, S. N. Brahmasandra et al., "An integrated nanoliter DNA analysis device," Science, vol. 282, no. 5388, pp. 484-487, 1998.

[21] F. Wang and M. A. Burns, "Performance of nanoliter-sized droplet-based microfluidic PCR," Biomedical Microdevices, vol. 11, no. 5, pp. 1071-1080, 2009.

[22] L. Shui, J. G. Bomer, M. Jin, E. T. Carlen, and A. van den Berg, "Microfluidic DNA fragmentation for on-chip genomic analysis," Nanotechnology, vol. 22, no. 49, Article ID 494013, 2011.

[23] J. Khandurina, T. E. McKnight, S. C. Jacobson, L. C. Waters, R. S. Foote, and J. M. Ramsey, "Integrated system for rapid PCR-based DNA analysis in microfluidic devices," Analytical Chemistry, vol. 72, no. 13, pp. 2995-3000, 2000.

[24] D. Sabourin, J. Petersen, D. Snakenborg et al., "Microfluidic DNA microarrays in PMMA chips: streamlined fabrication via simultaneous DNA immobilization and bonding activation by brief UV exposure," Biomedical Microdevices, vol. 12, no. 4, pp. 673-681, 2010.

[25] A. H. Diercks, A. Ozinsky, C. L. Hansen, J. M. Spotts, D. J. Rodriguez, and A. Aderem, "A microfluidic device for multiplexed protein detection in nano-liter volumes," Analytical Biochemistry, vol. 386, no. 1, pp. 30-35, 2009.

[26] K. F. Lei, "Quantitative electrical detection of immobilized protein using gold nanoparticles and gold enhancement on a biochip," Measurement Science and Technology, vol. 22, no. 10, Article ID 105802, 2011.

[27] K. F. Lei and Y. K. C. Butt, "Colorimetric immunoassay chip based on gold nanoparticles and gold enhancement," Microfluidics and Nanofluidics, vol. 8, no. 1, pp. 131-137, 2010.

[28] A. E. Herr, A. V. Hatch, D. J. Throckmorton et al., "Microfluidic immunoassays as rapid saliva-based clinical diagnostics," Proceedings of the National Academy of Sciences of the United States of America, vol. 104, no. 13, pp. 5268-5273, 2007.

[29] A. Bhattacharyya and C. M. Klapperich, "Design and testing of a disposable microfluidic chemiluminescent immunoassay for disease biomarkers in human serum samples," Biomedical Microdevices, vol. 9, no. 2, pp. 245-251, 2007.

[30] S. Lai, S. Wang, J. Luo, L. J. Lee, S. T. Yang, and M. J. Madou, "Design of a compact disk-like microfluidic platform for enzyme-linked immunosorbent assay," Analytical Chemistry, vol. 76, no. 7, pp. 1832-1837, 2004.
[31] D. Yang, X. Niu, Y. Liu et al., "Electrospun nanofibrous membranes: a novel solid substrate for microfluidic immunoassays for HIV," Advanced Materials, vol. 20, no. 24, pp. 4770-4775, 2008.

[32] T. Glawdel and C. L. Ren, "Electro-osmotic flow control for living cell analysis in microfluidic PDMS chips," Mechanics Research Communications, vol. 36, no. 1, pp. 75-81, 2009.

[33] F. T. G. van den Brink, E. Gool, J.-P. Frimat, J. Bomer, A. van den Berg, and S. le Gac, "Parallel single-cell analysis microfluidic platform," Electrophoresis, vol. 32, no. 22, pp. 3094-3100, 2011.

[34] R. N. Zare and S. Kim, "Microfluidic platforms for single-cell analysis," Annual Review of Biomedical Engineering, vol. 12, pp. 187-201, 2010.

[35] G. Mehta, J. Lee, W. Cha, Y.-C. Tung, J. J. Linderman, and S. Takayama, "Hard top soft bottom microfluidic devices for cell culture and chemical analysis," Analytical Chemistry, vol. 81, no. 10, pp. 3714-3722, 2009.

[36] K. F. Lei and P. H. M. Leung, "Microelectrode array biosensor for the detection of Legionella pneumophila," Microelectronic Engineering, vol. 91, pp. 174-177, 2012.

[37] K. F. Lei, M. H. Wu, C. W. Hsu, and Y. D. Chen, "Real-time and non-invasive impedimetric monitoring of cell proliferation and chemosensitivity in a perfusion $3 \mathrm{D}$ cell culture microfluidic chip," Biosensors and Bioelectronics, vol. 51, pp. 16-21, 2014.

[38] K. F. Lei, M. H. Wu, P. Y. Liao, Y. M. Chen, and T. M. Pan, "Development of a micro-scale perfusion 3D cell culture biochip with an incorporated electrical impedance measurement scheme for the quantification of cell number in a 3D cell culture construct," Microfluidics and Nanofluidics, vol. 12, no. 1-4, pp. 117-125, 2012.

[39] M. Bu, T. Melvin, G. Ensell, J. S. Wilkinson, and A. G. R. Evans, "Design and theoretical evaluation of a novel microfluidic device to be used for PCR," Journal of Micromechanics and Microengineering, vol. 13, pp. S125-S130, 2003.

[40] K.-S. Ma, H. Zhou, J. Zoval, and M. Madou, "DNA hybridization detection by label free versus impedance amplifying label with impedance spectroscopy," Sensors and Actuators B: Chemical, vol. 114, no. 1, pp. 58-64, 2006.

[41] S. J. Park, T. A. Taton, and C. A. Mirkin, "Array-based electrical detection of DNA with nanoparticle probes," Science, vol. 295, no. 5559, pp. 1503-1506, 2002.

[42] C. Fang, Y. Fan, J. Kong, Z. Gao, and N. Balasubramanian, "Electrical detection of oligonucleotide using an aggregate of gold nanoparticles as a conductive tag," Analytical Chemistry, vol. 80, no. 24, pp. 9387-9394, 2008.

[43] R. L. Testerman, N. R. Hagfors, and S. I. Schwartz, "Design and evaluation of nerve stimulating electrodes," Medical research engineering, vol. 10, no. 1, pp. 6-11, 1971.

[44] M. Sawan, F. Mounaim, and G. Lesbros, "Wireless monitoring of electrode-tissues interfaces for long term characterization," Analog Integrated Circuits and Signal Processing, vol. 55, no. 1, pp. 103-114, 2008.

[45] A. Yufera, G. Leger, E. O. Rodriguez-Villegas et al., "An integrated circuit for tissue impedance measure," in Proceedings of the 2nd Annual International IEEE-EMB Special Topic Conference on Microtechnologies in Medicine \& Biology, pp. 88-93, Madison, Wis, USA, 2002.

[46] A. Yúfera, A. Rueda, J. M. Muñoz, R. Doldán, G. Leger, and E. O. Rodríguez-Villegas, "A tissue impedance measurement chip for myocardial ischemia detection," IEEE Transactions on Circuits and Systems I: Regular Papers, vol. 52, no. 12, pp. 2620-2628, 2005. 
[47] R. Pallas-Areny and J. G. Webster, "Bioelectric impedance measurements using synchronous sampling," IEEE Transactions on Biomedical Engineering, vol. 40, no. 8, pp. 824-829, 1993.

[48] J. Sacristán-Riquelme, F. Segura-Quijano, A. Baldi, and M. Teresa Osés, "Low power impedance measurement integrated circuit for sensor applications," Microelectronics Journal, vol. 40, no. 1, pp. 177-184, 2009.

[49] A. Yúfera and A. Rueda, "Design of a CMOS closed-loop system with applications to bio-impedance measurements," Microelectronics Journal, vol. 41, no. 4, pp. 231-239, 2010.

[50] A. S. Sedra and K. C. Smith, Microelectronic Circuit, Oxford University Press, New York, NY, USA, 5th edition, 2007.

[51] A. Harb and M. Sawan, "New low-power low-voltage highCMRR CMOS instrumentation amplifier," in Proceedings of the IEEE International Symposium on Circuits and Systems (ISCAS '99), vol. 6, pp. 97-100, June 1999.

[52] C. C. Enz and G. C. Temes, "Circuit techniques for reducing the effects of Op-Amp imperfections: autozeroing, correlated double sampling, and chopper stabilization," Proceedings of the IEEE, vol. 84, no. 11, pp. 1584-1614, 1996.

[53] R. R. Harrison and C. Charles, "A low-power low-noise CMOS amplifier for neural recording applications," IEEE Journal of Solid-State Circuits, vol. 38, no. 6, pp. 958-965, 2003.

[54] J. A. Tierno, A. V. Rylyakov, and D. J. Friedman, "A wide power supply range, wide tuning range, all static cmos all digital pll in $65 \mathrm{~nm}$ SOI," IEEE Journal of Solid-State Circuits, vol. 43, pp. 42-51, 2008.

[55] C. S. Alex Gong, K. W. Yao, and M. T. Shiue, "A CMOS multichannel electrical stimulation prototype system," International Journal of Circuit Theory and Applications, vol. 41, no. 3, pp. 238$258,2013$.

[56] M. Ghovanloo and K. Najafi, "A wireless implantable multichannel microstimulating system-on-a-chip with modular architecture," IEEE Transactions on Neural Systems and Rehabilitation Engineering, vol. 15, no. 3, pp. 449-457, 2007.

[57] Y. Chen, C. C. Wong, T. S. Pui et al., "CMOS high density electrical impedance biosensor array for tumor cell detection," Sensors and Actuators B: Chemical, vol. 173, pp. 903-907, 2012.

[58] G. Lesbros and M. Sawan, "Multiparameters monitoring for long term in-vivo characterization of electrode-tissues contacts," in Proceedings of the 13th IEEE International Conference on Electronics, Circuits and Systems (ICECS '06), pp. 25-28, Nice, France, December 2006.

[59] C. S. A. Gong, K. W. Yao, M. T. Shiue, and Y. Chang, "An impedance measurement analog front end for wirelessly bioimplantable applications," in Proceedings of the IEEE Asia Pacific Conference on Circuits and Systems (APCCAS '12), pp. 172-175, Kaohsiung, Taiwan, December 2012.

[60] I. Bantounas, L. A. Phylactou, and J. B. Uney, "RNA interference and the use of small interfering RNA to study gene function in mammalian systems," Journal of Molecular Endocrinology, vol. 33, no. 3, pp. 545-557, 2004.

[61] Y. A. Abassi, B. Xi, W. Zhang et al., "Kinetic cell-based morphological screening: prediction of mechanism of compound action and off-target effects," Chemistry and Biology, vol. 16, no. 7, pp. 712-723, 2009.

[62] "Using the xCELLigence System for Functional Genomics: Assessing Gene Function in Real Time," Application Note of ACEA Biosciences, Inc. 


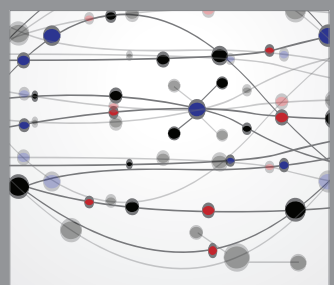

The Scientific World Journal
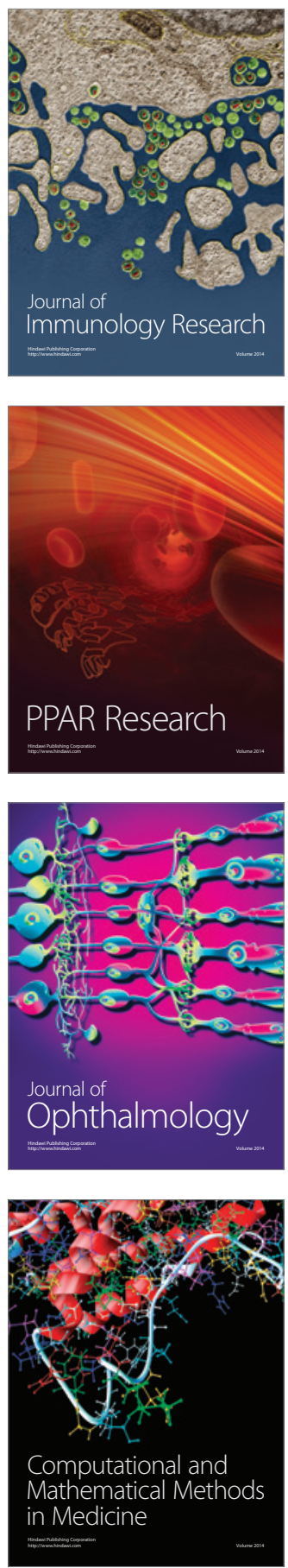

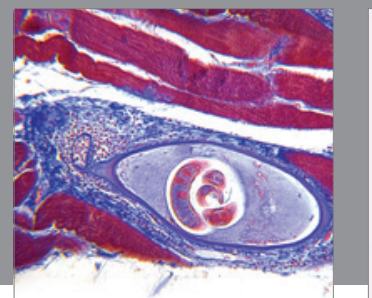

Gastroenterology

Research and Practice
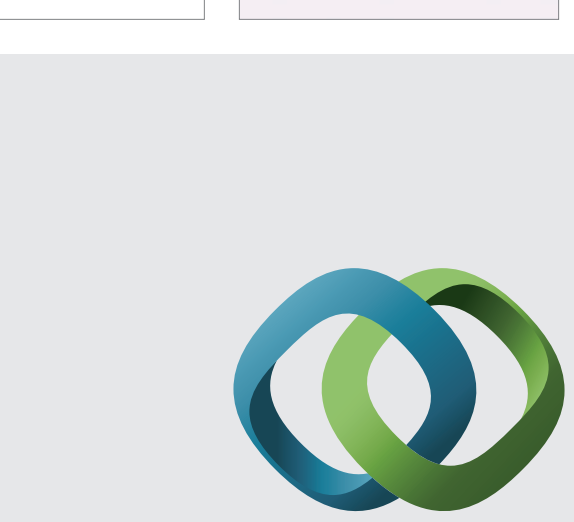

\section{Hindawi}

Submit your manuscripts at

http://www.hindawi.com
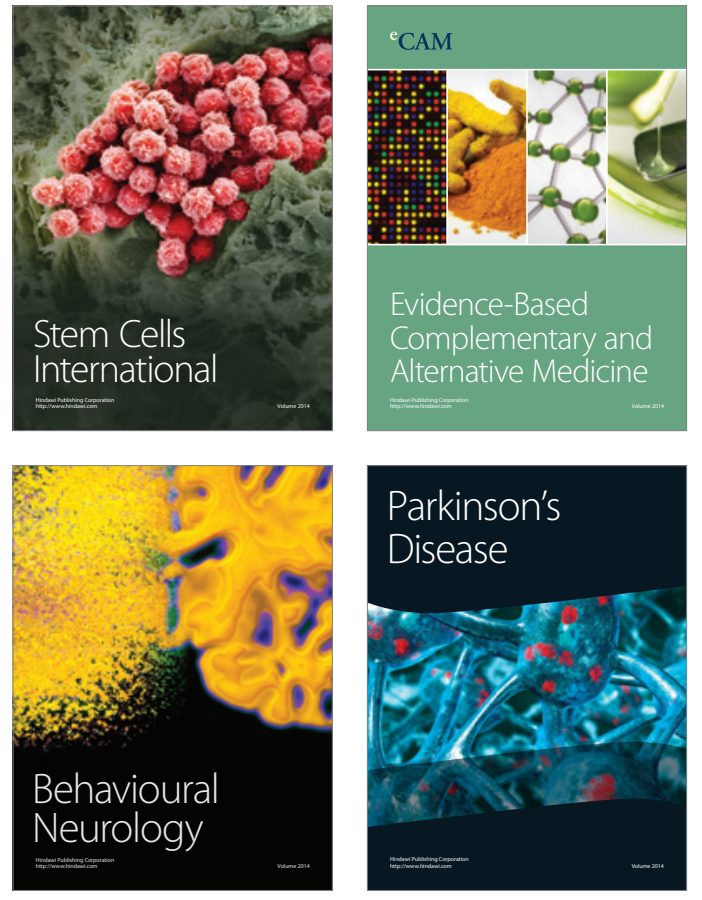
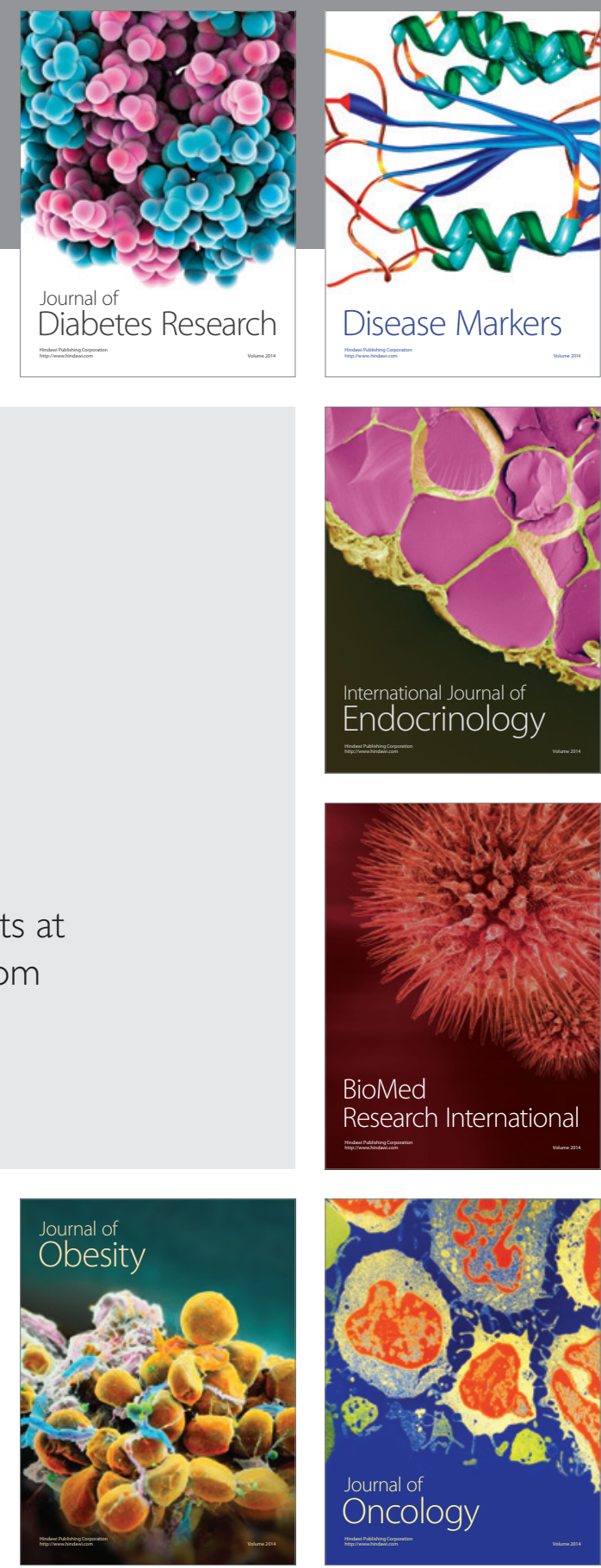

Disease Markers
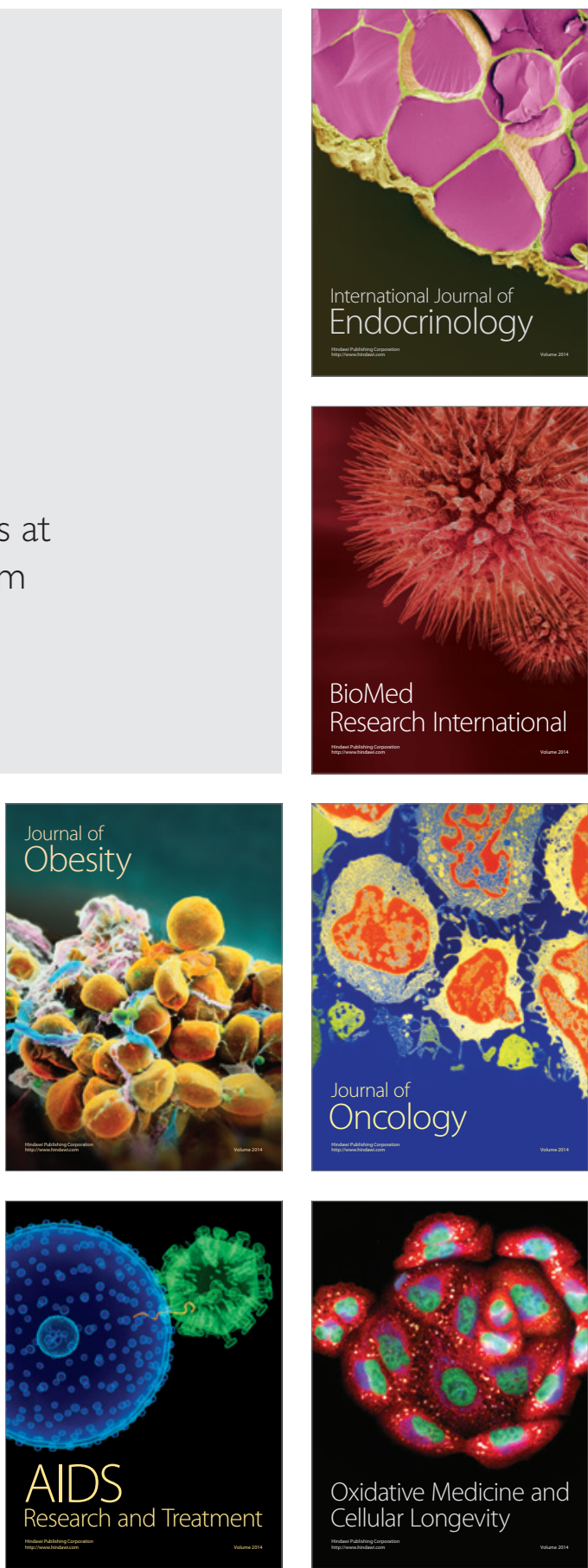